\title{
A Comparison of the Morphology and Stability of Relativistic and Nonrelativistic Jets
}

\author{
Alexander Rosen ${ }^{1}$, rosen@eclipse.astr.ua.edu, \\ Philip A. Hughes² ${ }^{2}$ hughes@astro.lsa.umich.edu, \\ G. Comer Duncan³ ${ }^{3}$ gcd@chandra.bgsu.edu, \\ and Philip E. Hardee ${ }^{1}$, hardee@venus.astr.ua.edu \\ ${ }^{1}$ Department of Physics and Astronomy, University of Alabama, Tuscaloosa, AL 35487 \\ ${ }^{2}$ Astronomy Department, University of Michigan, Ann Arbor, MI 41809 \\ ${ }^{3}$ Department of Physics and Astronomy, Bowling Green State University, Bowling Green, \\ OH 43403 \\ Accepted for publication by The Astrophysical Journal \\ To appear in the May 10, 1999 issue
}

\begin{abstract}
We compare results from a relativistic and a nonrelativistic set of $2 \mathrm{D}$ axisymmetric jet simulations. For a set of five relativistic simulations that either increase the Lorentz factor or decrease the adiabatic index we compute nonrelativistic simulations with equal useful power or thrust. We examine these simulations for morphological and dynamical differences, focusing on the velocity field, the width of the cocoon, the age of the jets, and the internal structure of the jet itself.

The primary result of these comparisons is that the velocity field of nonrelativistic jet simulations cannot be scaled up to give the spatial distribution of Lorentz factors seen in relativistic simulations. Since the local Lorentz factor plays a major role in determining the total intensity for parsec scale extragalactic jets, this suggests that a nonrelativistic simulation cannot yield the proper intensity distribution for a relativistic jet. Another general result is that each relativistic jet and its nonrelativistic equivalents have similar ages (in dynamical time units, $\equiv \mathrm{R} / a_{\mathrm{a}}$, where $\mathrm{R}$ is the initial radius of a cylindrical jet and $a_{\mathrm{a}}$ is the sound speed in the ambient medium). Also, jets with a larger Lorentz factor have a smaller cocoon size.
\end{abstract}


In addition to these comparisons, we have completed four new relativistic simulations to investigate the effect of varying thermal pressure on relativistic jets. The simulations confirm that faster (larger Lorentz factor) and colder jets are more stable, with smaller amplitude and longer wavelength internal variations. However, an exception to this occurs for the hottest jets, which appear the most stable. The apparent stability of these jets does not follow from linear normal mode analysis, which suggests that there are available growing Kelvin-Helmholtz modes. However, these modes are not excited, due to a lack of perturbations able to couple to them.

As an example of how these simulations can be applied to the interpretation of observations, we use our results to estimate some parameters of Cygnus A. Although none of these estimates alone can determine if the jets in Cyg A are relativistic or nonrelativistic, estimates for the age and the jet-to-ambient density ratio confirm values for these parameters estimated by other means.

Subject headings: galaxies : individual (Cyg A) — galaxies : jets hydrodynamics — instabilities — relativity

\section{Introduction}

Jet flows in extragalactic radio sources and those associated with the Galactic superluminals could have relativistic velocities for a significant fraction of their length (for example, for extragalactic sources see the arguments in Cawthorne 1991, and for Galactic superluminals see the introduction in Hardee et al. 1998, hereafter HRHD). Recently, a number of groups have begun to simulate such relativistic flows (Duncan \& Hughes 1994, hereafter DH94; Falle \& Komissarov 1996; Komissarov \& Falle 1997; Martí et al. 1995; and Martí et al. 1997). There have even been preliminary investigations of relativistic magnetohydrodynamics (Koide, Nishikawa, \& Mutel 1996; van Putten 1996), including one attempt at combining MHD with general relativity in $3+1$ dimensions (Koide, Shibata \& Kudoh 1998). While these papers and a review (Norman 1996) have discussed some of the qualitative differences between relativistic and nonrelativistic jets, a more thorough comparison is essential in order to understand whether nonrelativistic simulations can be applied to the interpretation of observed relativistic flows, and whether a comparison of nonrelativistic and relativistic simulations can provide a diagnostic of flow speed.

Many researchers have performed simulations of nonrelativistic jets over the last 10-15

years, with reviews of the early work in Norman, Winkler, \& Smarr (1984) and Norman \& 
Winkler (1985). Investigations frequently focused on the question of jet disruption by fluid instabilities, and in this light Norman, Winkler, \& Smarr (1984) discuss the susceptibility of the jet to the ordinary or fundamental and reflecting modes of the Kelvin-Helmholtz (hereafter $\mathrm{KH}$ ) pinch axisymmetric instability. Much recent literature uses the terms surface wave and body wave for the ordinary and reflecting mode, respectively, and we adopt that here. For each of the pinch, helical, triangular, rectangular and higher order normal KH modes there is one surface and many body waves. Axisymmetric simulations, like the ones discussed here, only allow waves of the pinch mode, since higher order modes require non-axisymmetry. Note that the higher order modes have smaller growth lengths (or faster growth rates) than the pinch mode, so axisymmetric jets remain essentially stable for longer distances than in simulations where higher order modes are allowed (e.g., a 2D slab jet simulation: Hardee \& Norman 1988; Bodo et al. 1994; Bodo et al. 1995; or any 3D jet simulation: e.g., Clarke 1996). Another reason for expecting that the flows studied here will exhibit little instability is that previous analytic and numerical work has shown that jets with relativistic flow speeds are more stable than jets with slower flow speeds (HRHD; Martí et al. 1997), primarily because the former jets behave as if they are more dense.

In this paper we will compare 2D axisymmetric nonrelativistic jet simulations performed with ZEUS-3D and simulations performed with a relativistic Godunov (shock-capturing) scheme discussed in DH94. For each relativistic simulation we have computed nonrelativistic equivalents as suggested by Komissarov (1996), based on equating the useful power (i.e., the enthalpy flux minus the mass-energy flux) or the thrust of the jet at the inlet. We show that one of these sets of nonrelativistic equivalents should have stability properties (i.e., spatial growth rates, etc.) that are similar to those of many of the relativistic simulations. These similar stability properties will lead to similar structures within the jet: for example, small-scale features along the jet axis that may grow to become shocks.

Also, we investigate the effect of jet temperature on the structure of the relativistic simulations. As opposed to the morphology of nonrelativistic jets, which primarily depends on jet-to-ambient density and Mach number (assuming that jet and ambient thermal pressures are equal; Norman, Winkler, \& Smarr 1984), the morphology of relativistic jets depends additionally on temperature as an independent parameter. One natural consequence of a "hot" relativistic jet, where the thermal velocities of particles are at least mildly relativistic, is that the classical or Newtonian Mach number $\left(\equiv v / a_{\mathrm{j}}\right.$, where $v$ is the jet velocity relative to the laboratory frame and $a_{\mathrm{j}}$ is the sound speed within the jet; hereafter we refer to this as the "Mach number") is small enough that the flow is transonic (with a Mach number between one and three). However, linear stability analysis suggests that the spatial growth length of the KH instability is roughly proportional to the product of the Lorentz factor and Mach number, and not the Mach number alone (HRHD). Thus, 
a hot relativistic flow with low Mach number should be less prone to instability, although still not completely stable, than the Mach number alone would indicate. In fact, Martí et al. (1997) see this relative stability in their hot jets, which have fewer internal features. However, their simulations spanned a wide range of specific internal energies with hot jets quite hot and cold jets quite cold. We have simulated jets with a similar range, 3-4 orders of magnitude, of specific internal energies, but with more resolution in temperature space and with specific internal energy density in some cases closer to unity. We also wish to reiterate the main point of HRHD: that relativistic jets are not unconditionally stable many simulations that have been computed contain perturbations that only weakly excite any of the available growing modes.

In $\S 2$, we describe the simulations with particular emphasis on the nonrelativistic equivalents, in $\S 3$, we discuss the various results while focusing on large scale structure and the growth of the $\mathrm{KH}$ instability, and in $\S 4$, we use some of the analysis presented here to make estimates of some fundamental quantities (e.g., the age, jet-to-external ratios for density and enthalpy, and the Mach number) for the jets in Cygnus A, a well-studied radio source. In addition, we include two Appendices; in Appendix A we derive the relativistic form of the jet head advance speed and in Appendix B we develop a simple model to estimate the cocoon radius in either relativistic or nonrelativistic jets.

\section{Simulations}

For the nonrelativistic simulations, we used a cylindrical coordinate system and the same boundary conditions as did DH94. Specifically, the boundary conditions allow outflow everywhere with two exceptions: at the inlet with inflow boundary conditions and along the symmetry axis, which has a reflecting boundary. DH94 describe a relativistic code that uses Adaptive Mesh Refinement (AMR), a general purpose scheme written by Quirk (1991), to gain spatial and temporal resolution. The simulations reported in DH94 employed one level of refinement: the coarse grid had 6 zones/jet radius and the refined grid had 24 zones/jet radius. We have run the nonrelativistic simulations using ZEUS-3D with a resolution of 12 zones/jet radius and the van Leer linear advection scheme, which is a combination that resolves features of the same size as the relativistic code, as determined by examining schlieren (density gradient) plots. The grid extends to $41^{2} / 3$ jet radii along the jet axis and either $10 \mathrm{R}$ or $16^{2} / 3 \mathrm{R}$ radially, depending on whether the relevant relativistic simulation had a Lorentz factor below or above 5, respectively. The nonrelativistic simulations were run on grids of either $500 \times 120$ or $500 \times 200$ zones.

The relativistic simulations are as described in DH94. In order to fill a gap in the 
DH94 simulations at small Lorentz factors, we include an additional relativistic simulation that has a Lorentz factor of 2.5 and was labeled Run E in HRHD. Run E fits between Runs $\mathrm{A}$ and B in the original DH94 sequence. We point out that HRHD adopted incorrect values for the Lorentz factor when analyzing Runs $\mathrm{E}$ (2.55 instead of 2.5), B (5.5 instead of 5.0) and $\mathrm{C}$ (14.35 instead of 10.0) and that we use the correct values here. Since the correct Lorentz factors reduce by $\lesssim 30 \%$ the maximum allowable wavelengths and the wavelengths associated with the fastest growth in the most important modes in Runs B and C, this correction does not alter significantly either the specific results or the overall conclusions in HRHD.

For the relativistic simulations this paper generally uses the notation of DH94; $n$ is the proper mass density, $c$ is the speed of light, $\beta$ is $v / c, \gamma$ is the Lorentz factor $\left(\gamma \equiv\left(1-\beta^{2}\right)^{-1 / 2}\right)$, $\Gamma$ is the adiabatic index, $p$ is the internal (or thermal) pressure, $e$ is the proper mass-energy density and is $e=n c^{2}+p /(\Gamma-1)$, and the enthalpy is $e+p=n c^{2}+(\Gamma p /(\Gamma-1))$. One change from DH94 is that the mass density in the lab frame for relativistic simulations is designated $\nu$ and not $\mathrm{R}$, which we use for the initial jet radius. In this paper, we designate the jet-to-ambient density ratio as $\eta$, and the jet-to-ambient enthalpy ratio as $\eta_{\mathrm{r}}$. We will discuss $\eta$ primarily in the nonrelativistic simulations, but where we specifically use $\eta$ in regard to the relativistic simulations, this refers the ratio of proper densities and is 0.1 for all of our relativistic simulations. We use the variable $\rho$ exclusively for the mass density in the nonrelativistic simulations. Additionally, when comparing a similar quantity between relativistic and nonrelativistic simulations, we occasionally refer to each with rel or non subscripts.

For the nonrelativistic simulations, we need specify only two parameters, assuming that the jet and ambient thermal pressures are equal, as in the simulations of DH94. The morphology of 2D axisymmetric nonrelativistic jets depends on only $\eta$ and M, the Mach number with respect to the sound speed within the jet (Norman, Winkler, \& Smarr 1984). In this paper, Mach number always refers to this "internal" value and we will refer to the Mach number with respect to the ambient or external medium as either $\mathrm{M}_{\mathrm{a}}\left(\equiv v / a_{\mathrm{a}}\right)$ or $\mathrm{M}_{\mathrm{x}}$ $\left(\equiv v / a_{\mathrm{x}}\right)$, respectively. Here, the ambient medium refers to the fluid into which the jet is propagating and the external medium refers to the fluid in contact with the length of the jet, which can be either the shocked ambient medium or the cocoon and is important for the development of the KH instability. In jets with flow speeds that are relativistic, there is a quantity analogous to the Mach number, the relativistic or proper Mach number, $\mathcal{M}$ $\equiv \gamma \beta / \gamma_{\mathrm{s}} \beta_{\mathrm{s}}$, as defined in the discussion of time-independent relativistic flows by König (1980); the subscript $s$ refers to the sound speed (e.g., $\beta_{\mathrm{s}} \equiv a_{\mathrm{j}} / c$ ). Since the nonrelativistic simulations are scale-free and $\eta$ and $M$ are the main independent variables, the ambient medium density and sound speed are set initially to unity in the ZEUS code. 
We will discuss two sets of nonrelativistic equivalents, both of which are suggested by Komissarov (1996). One can determine $\eta$ and $\mathrm{M}$ for a nonrelativistic simulation by assuming that the velocities and pressures are equal in a relativistic simulation and its nonrelativistic equivalent and equating one of the following fluxes: useful power, thrust, or mass. We feel that the mass flux equivalent is less interesting, because the mass flux is physically less important than the other two fluxes and quantities that are associated with the mass flux, such as the mass of a radio lobe, are rarely estimated from observational data. Thus, we have chosen not to compute the mass flux equivalent simulations. Equating the momentum flux of jets in the two types of simulations leads to a more interesting situation. Since momentum flux and ram pressure are equivalent within either the relativistic or nonrelativistic formulation, there is a similar expected jet-head-to-jet velocity ratio in both a relativistic simulation and its nonrelativistic momentum flux equivalent. Another interesting case involves the useful power $\left(\equiv \gamma^{2}(e+p) v-\gamma n c^{2} v\right.$ in the relativistic simulations), which is the energy flux that feeds the observed luminosity of radio lobes.

For the useful power and thrust equating cases, we list below the equivalent nonrelativistic mass density and Mach number in terms of the relativistic fluid variables for each region of the flow. Recall that $\eta=\rho_{\mathrm{j}} / \rho_{\mathrm{a}}$ and thus we need to calculate the equivalent nonrelativistic mass density in both the jet and ambient medium. The relationships for $\rho$ and $\mathrm{M}$ in the useful power equivalent are (Komissarov 1996):

$$
\begin{gathered}
\rho=2 n \gamma^{2}\left(\frac{\gamma}{\gamma+1}+\frac{\Gamma p}{(\Gamma-1) n c^{2}}\right) \\
\text { and } \quad \mathrm{M}^{2}=2 \mathcal{M}^{2}\left[\frac{\left(\frac{\gamma}{\gamma+1}+\frac{\Gamma}{(\Gamma-1)} \frac{p}{n c^{2}}\right)}{\left(1+\frac{\Gamma(2-\Gamma)}{\Gamma-1} \frac{p}{n c^{2}}\right)}\right] .
\end{gathered}
$$

The relationships that follow from equating thrust are:

$$
\begin{gathered}
\rho=\gamma^{2} n\left(1+\frac{\Gamma}{\Gamma-1} \frac{p}{n c^{2}}\right) \\
\text { and } \quad \mathrm{M}^{2}=\left(\gamma^{2}-1\right)\left(\frac{n c^{2}}{\Gamma p}+\frac{1}{(\Gamma-1)}\right) .
\end{gathered}
$$

Note that equation (4) reduces to $\mathrm{M}_{\text {non }}=\gamma \mathrm{M}_{\text {rel }}$. Of the relativistic simulations, the pressure in the ambient medium is most significant in Runs $\mathrm{C}$ and $\mathrm{D}$, increasing the equivalent $\rho$ and decreasing $\eta$ by a factor of three (for useful power) or two (for thrust). 
We will refer to each of the nonrelativistic equivalents by the relativistic simulation to which they are similar and two letters describing the nature of the equivalence, e.g., $\mathrm{B}_{\mathrm{pw}}$ and $\mathrm{B}_{\mathrm{th}}$ for the useful power and thrust equivalent, respectively, for the relativistic simulation Run B. Since we computed only one nonrelativistic equivalent of Run A, we will refer to this as Run $\mathrm{A}_{\mathrm{pw}}$.

The values of $\eta$, internal Mach number $(M)$ and ambient Mach number $\left(M_{a}\right)$ are shown in Table 1 for both sets of nonrelativistic simulations. Note that Run D in DH94 used a softer adiabatic index, $\Gamma=4 / 3$, for the jet and ambient medium, and that we have reproduced this in the nonrelativistic equivalents of Run D. Since our relativistic simulations have the same $\eta$, for these simulations we list the enthalpy ratio $\eta_{\mathrm{r}}$, which is one measure of relativistic thermal motions, in Table 1 . The progression of $\eta_{\mathrm{r}}$ is related to the increase in $p$ as $\gamma$ is increased in the set of relativistic jets. Since these simulations model light jets pressure-matched with the ambient medium, increasing $p$ leads to $\eta_{\mathrm{r}}$ asymptotically approaching unity from below.

The nonrelativistic equivalent jets are frequently denser than the external medium, which is a consequence of relativistic jets behaving as if they have a higher density than their proper density. Specifically, $\eta$ would have to be $\lesssim 10^{-3}$ in the most relativistic simulations (Runs C and D) for nonrelativistic equivalents to have $\eta<1$. Examining the values of $\mathrm{M}_{\mathrm{a}}$ reveals that the most relativistic simulations have power- and thrust-equating nonrelativistic equivalents that are transonic $\left(1 \lesssim \mathrm{M}_{\mathrm{a}} \lesssim 3\right)$ with respect to the ambient medium.

One goal of this analysis is to compare the analytically predicted growth of the $\mathrm{KH}$ instability modes with the internal structure found in the two sets of simulations. We can derive Mach numbers and jet-to-external (i.e., cocoon, not ambient) density ratios for a nonrelativistic jet with stability properties similar to those of a relativistic jet by considering the approximation for the wave speed of $\mathrm{KH}$-induced disturbances at resonance (or maximum growth), $v_{\mathrm{w}}^{*}$, for a relativistic cylindrical jet (from HRHD, equation 6c):

$$
v_{\mathrm{w}}^{*} \approx \frac{\gamma\left[\mathrm{M}^{2}-\beta^{2}\right]^{1 / 2}}{\left[\mathrm{M}_{\mathrm{x}}^{2}-\beta^{2}\right]^{1 / 2}+\gamma\left[\mathrm{M}^{2}-\beta^{2}\right]^{1 / 2}} v .
$$

The product $\gamma \mathrm{M}$ in relativistic jets appears approximately in the same role as the Mach number in nonrelativistic jets, and is the same form used for $\mathrm{M}$ in the thrust-equating case. In order to estimate the jet-to-external density ratio, $\eta_{\mathrm{x}}$, we take the nonrelativistic limit for $v_{\mathrm{w}}^{*} / v: \mathrm{M} /\left(\mathrm{M}_{\mathrm{x}}+\mathrm{M}\right)$. Assuming that the jet and external pressures are equal, this term reduces to $\sqrt{\eta_{\mathrm{x}}} /\left(\sqrt{\eta_{\mathrm{x}}}+1\right)$. Using values of $\beta, \mathrm{M}$, and $\mathrm{M}_{\mathrm{x}}$ from the relativistic simulations, we solve for desired values of $\eta_{\mathrm{x}}$ in the nonrelativistic simulations by equating $v_{\mathrm{w}}^{*} / v$ in eq. (5) to its nonrelativistic limit. Note that typically $M_{x} \neq M_{a}$, but that we do list $M$ and 
$\mathrm{M}_{\mathrm{x}}$ for Runs $\mathrm{E}, \mathrm{B}$, and $\mathrm{C}$ below. For the nonrelativistic equivalents of all but relativistic Run A, $\eta_{\mathrm{x}}$ corresponds to an overdense jet (see Table 1), dense enough that there is no significant difference between $\eta_{\mathrm{x}}$ and $\eta$. Additionally, these values are roughly similar to the set of density ratios used in the thrust-equating simulations, which thus have both independent parameters appropriate for matching $\mathrm{KH}$ stability properties. Even though Runs $\mathrm{E}_{\mathrm{th}}$ and $\mathrm{B}_{\mathrm{th}}$ have density ratios quite different from the desired $\eta_{\mathrm{x}}$, the density ratios in Runs $\mathrm{C}_{\text {th }}$ and $\mathrm{D}_{\text {th }}$ are a closer match. Also, note that the $\eta$ in Run $\mathrm{B}_{\text {th }}$ is similar to the desired $\eta_{\mathrm{x}}$ for Run $\mathrm{E}$, with only a small difference in Mach number between $\mathrm{E}_{\mathrm{th}}$ and $\mathrm{B}_{\mathrm{th}}$.

This suggests that the jet in Run $B_{\text {th }}$ should have similar stability properties as those of the jet in relativistic Run E.

\section{Results}

We show schlieren plots, in which darker regions indicate a larger density gradient, for all of the simulations as the bow shock nears the right edge of the grid $(z \sim 40 \mathrm{R})$ in Figure 1. Typical jet features include a bow shock, a terminal shock or Mach disk of the jet flow, a cocoon of material lighter than the jet that has passed through this shock, and internal structure in the form of biconical shocks along the jet. In the smaller Lorentz factor relativistic simulations, there is some curvature of the terminal shock, which is the very thin line close to the leading edge of the jet and almost perpendicular to the jet axis in the schlieren images. There is some difference in this curvature between the simulations, particularly between Runs E and B, and in general the shape and curvature of the terminal shock varies with time. In some of the simulations and in particular Run $\mathrm{C}$, there is a secondary shock roughly 10-20R behind and parallel to the bow shock, which is caused by shocked ambient material expanding supersonically backwards. This secondary shock is weaker, or nonexistent in Run D, a consequence of slower expansion accompanying the softer adiabatic index. Some of the schlieren plots show a nonphysical reflection of the bow shock at the outer radial boundary, generated by the edge of the numerical grid. The strength of this feature is somewhat exaggerated in a schlieren plot and the reflected bow shock does not interact significantly with any of the more important jet structures. Despite the appearance that the reflection coincides with an enhancement in cocoon width in Run $\mathrm{B}$, we have determined that these coincident structures are unrelated. In this case, the expansion of the cocoon occurs before the reflection arrives.

From the schlieren images in Figure 1, we see that the morphology of the jets changes with Lorentz factor in a similar manner for all three sets of simulations. However, the two codes do not give identical results for the relativistic simulation with a nonrelativistic jet 
flow speed and for its nonrelativistic equivalent, i.e. Runs $A$ and $A_{p w}$. While the cocoon width is similar, the detailed structure of the cocoon with the relativistic code is more regular and appears somewhat underesolved. This is caused by the relatively poor capturing of contact discontinuities by the Relativistic Harten-Lax-van Leer-Einfeldt (RHLLE, see Einfeldt 1988 and DH94) solver used in the relativistic simulations. These differences within the cocoon lead to the less regularly spaced biconical shocks within the jet in the nonrelativistic equivalent (Run $A_{p w}$ ). Given these differences between the numerical schemes for the same simulation, we will focus primarily on large-scale differences such as in the cocoon, jet head, and bow shock between a relativistic simulation and its nonrelativistic equivalents.

\subsection{Cocoon}

\subsubsection{Morphological Differences}

With the aim of examining any differences in the appearance between relativistic simulations and their nonrelativistic equivalents and since in the nonrelativistic simulations we can rescale the velocity field, we do so by equating inlet velocities for a relativistic simulation and its nonrelativistic equivalent. From the rescaled $\beta$ in each zone in the nonrelativistic simulations, we compute a scaled Lorentz factor. Doppler boosting is to first order the primary contributor to the observed intensity from the flow. Because Doppler boosting dominates variations in the inferred intrinsic emissivity, for a given angle of view the Lorentz factor should provide a useful (if coarse) estimator of the appearance of the jets at radio wavelengths.

We display contours of two values of Lorentz factor for the relativistic simulations and for the same two values of scaled Lorentz factor for the useful power set of nonrelativistic simulations in Figure 2. The adopted contours in these plots are at a low Lorentz factor, which is near unity but different for each simulation (see Fig. 2 for the values), and at the inflow Lorentz factor in the appropriate relativistic simulation. Assuming that the fluid flow is along the jet and that an observer is viewing the source close to the critical cone (with a viewing angle $\approx 1 / \gamma$ ), the radial width of these contours suggests an effective radius of observed intensity for the flow. Since we will not compare simulated intensity within any set of simulations, assuming different viewing angles for each relativistic simulation does not invalidate the following analysis. We show only the useful power nonrelativistic equivalents in Figure 2 because the thrust-equated set has a similar appearance.

Since nonrelativistic hydrodynamics imposes no upper bound on velocity, rescaled 
velocities above $c$ are unavoidable. Rescaled $\beta$ s above unity occur in most of the nonrelativistic equivalents of Runs $\mathrm{C}$ and $\mathrm{D}$ (the only exception of the four cases is Run $\mathrm{D}_{\mathrm{pw}}$ ), although the maximum rescaled $\beta$ in any simulation is 1.02 in Run $\mathrm{C}_{\mathrm{th}}$. Therefore, these equivalents are able to accommodate velocity rescaling reasonably well. The maxima of the rescaled $\beta$ is smaller for equivalents of Run D than for those of Run C. Correspondingly, the maximum speed in relativistic Run D is smaller than the maximum speed in relativistic Run C; one effect of a softer adiabatic index is to reduce the acceleration of the jet.

In general, the relativistic simulations have a much larger width as measured by these Lorentz factor contours than do the nonrelativistic simulations. In Runs A and E, there are only small regions of high $\gamma$ along the jet axis. This is also true for Run $A_{p w}$, but not for Run $\mathrm{E}_{\mathrm{pw}}$. This similarity between Run A and $\mathrm{A}_{\mathrm{pw}}$ suggests that the different numerical methods admit similar accelerations in the simulations that are most similar (note that a $\gamma$ of 1.05 represents an acceleration of less than $2 \%$ above the initial jet velocity in Run A), even though the appearance differs considerably in detail. While some aspects of the contours in Runs $\mathrm{A}$ and $\mathrm{A}_{\mathrm{pw}}$ are similar, the most similar contours of any relativistic-nonrelativistic pair shown in Figure 2 are the contours in Runs $\mathrm{D}$ and $\mathrm{D}_{\mathrm{pw}}$, which are narrow over most of the jet length and only display a small cocoon-like bulge near the terminal shock. Also, noticeable in the high- $\gamma$ contour is the pressure wave close to the inlet, which was discussed in HRHD (the contour starts at $r \sim \mathrm{R}$ near the inlet and moves inward farther along the jet axis). This pressure wave is easiest to see in Runs $\mathrm{C}, \mathrm{D}$, and $\mathrm{D}_{\mathrm{pw}}$, and is perhaps seen in Runs $\mathrm{B}$ and $\mathrm{E}_{\mathrm{pw}}$ as well.

Given our assumption that there is a correspondence between the Lorentz factor and the perceived radio intensity from simulated jets, we see from Figure 2 that the shape of emission regions are quite different between the pairs of simulations. Specifically, high Lorentz factor or "bright" regions are smaller near the jet head and the internal structure is more varied in the nonrelativistic equivalents than in their relativistic counterparts. These differences between relativistic and nonrelativistic equivalents may also be seen in lighter jets (Komissarov \& Falle 1996), but there the cocoon in the relativistic simulation contains significant backflow, suggesting that the cocoon could be Doppler enfeebled and much dimmer than the jet flow if the jet is directed toward the observer. The Doppler enfeeblement of radiation from a cocoon with a significant backflow could dim the cocoon by a large enough amount that light relativistic jets appear very narrow when the jet is directed nearly toward the observer. This narrow appearance of the cocoon and jet from a relativistic flow could reduce the difference between the apparent lobe widths of relativistic jets and their nonrelativistic equivalents. 
In both relativistic and nonrelativistic sets shown in Figure 2, the simulations with the most contours crossing the jet axis are for jets that have an underlying flow with a moderate $\gamma$, between roughly 2 and 5. Although this comparison only shows contours of $\gamma$ and is not a true representation of the total intensity from a radio source, this is highly suggestive that jet flows of moderate Lorentz factor may lead to the most "knotty" appearance, which confirms a result of Mioduszewski, Hughes, \& Duncan (1997).

We conclude that, despite the modest success of predicting the maximum flow velocity when rescaling a nonrelativistic simulation to the relativistic inflow speed, the rescaled velocities underscore the necessity of performing fully relativistic hydrodynamic simulations: the entire velocity field computed by a nonrelativistic simulation cannot be simply rescaled to yield the velocity field of a relativistic jet.

\subsubsection{Comparison with Simple Models}

By comparing the width of the cocoon seen in simulations with analytic estimates based on simple assumptions, we determine the viability of such an analytic model for the interpretation of data, and gain insight into the origin of different flow speed-dependent morphologies. We derive equations for the ratio of cocoon to jet radius $\left(R_{c} / R\right)$ for each of the relativistic and nonrelativistic cases in Appendix B. From these equations, we estimate a cocoon radius based on the relativistic form (equation [6]) for each of the relativistic simulations. While this relation is most applicable to Run D, because the derivation assumes $\Gamma=4 / 3$, we expect that the different adiabatic index in Runs A, E, B, and C will have little effect on the cocoon radius and that it is valid to generalize this simple model to include other adiabatic indices. This follows from the results of Duncan, Hughes, \& Opperman (1996), who recomputed Run C using a modified version of the relativistic hydrodynamic code that allowed for a variable adiabatic index. The recomputed simulation demonstrated that even though the simulation displayed significant spatial and temporal variations in $\Gamma$, there were minimal differences in the flow morphology.

We display contours of $R_{c} / R$ in Figure 3 from the analytic model in $\eta_{\mathrm{r}}-\gamma$ space for the relativistic case from Eq. 6 (derived in Appendix B.1) and in $\eta-\mathrm{M}$ space for the nonrelativistic case from Eq. 7 (derived in Appendix B.3):

$$
\left(\frac{R_{\mathrm{c}}}{R_{\mathrm{j}}}\right)^{2}=1+\left(\frac{p_{\mathrm{h}}}{p_{\mathrm{j}}}\right)^{1 / \Gamma} \frac{n_{\mathrm{j}}}{n_{\mathrm{h}}}\left(\frac{1+\eta_{\mathrm{r}}+2 \sqrt{\eta_{\mathrm{r}}} \gamma_{\mathrm{j}}}{\eta_{\mathrm{r}}}\right)^{1 / 2}
$$




$$
\left(\frac{R_{\mathrm{c}}}{R_{\mathrm{j}}}\right)^{2}=1+\left(1+\eta^{-1 / 2}\right) \frac{\rho_{\mathrm{j}}}{\rho_{\mathrm{h}}}\left(\frac{p_{\mathrm{h}}}{p_{\mathrm{j}}}\right)^{1 / \Gamma}
$$

For both cases, we show the positions of the appropriate simulations in parameter space. We have not placed the nonrelativistic equivalents for Run D in Fig. 3b, since the contours are shown for $\Gamma=5 / 3$. However, there is only a small difference between the expected cocoon radius between the two adiabatic indices, with the difference being less than $20 \%$ for any of our simulations.

We display the theoretical values of $R_{c} / R$ for each simulation as vertical lines in plots of radial profiles of density and pressure (Figure 4), which have been measured at the position of local minimum density that is indicated by an arrow in each of the schlieren images in Figure 1. In the panels that show data from a relativistic simulation, both the lab frame density and proper density radial profiles are shown. In all three simulation sets, the cocoon radius expected from the simple model typically decreases as the Lorentz factor is increased, with the exception of both nonrelativistic equivalents of Run C, which have an expected $\mathrm{R}_{\mathrm{c}}$ slightly larger than that of each Run B equivalent. Frequently the theoretical values in Figure 4 are coincident with a local minimum in density or pressure, usually well within the cocoon, which we interpret as the cocoon center. The pressure usually increases outward from this radial position, consistent with a nearly fully expanded cocoon. While all three data profiles typically are similar within each relativistic simulation, there is one exception. The expected $\mathrm{R}_{\mathrm{c}}$ in Run $\mathrm{C}$ occurs at a smaller radius than the position of the minimum proper density and a larger radius than the position of the minimum lab frame density, but it is well-centered within a wide minimum in pressure. Thus, the analytic estimate of $R_{c}$ is a better indicator of the radial position of minimum pressure. Also, this estimate is closer to local extrema in the relativistic cases than in the nonrelativistic simulations. Specifically, in the nonrelativistic equivalents of Runs C and D, the expected $R_{c}$ is within the shocked ambient medium or farther from the jet axis than any cocoon-like feature in the radial profile.

Some of the underlying assumptions for the analytic expressions in Appendix B are demonstrably poor. Specifically, two of these assumptions are unlikely to be satisfied: 1) pressure balance between the jet, cocoon, and the ambient medium and 2) a slow advance speed of the terminal shock in the lab frame. The radial profiles in Figure 4 show that the variation of pressure is typically an order of magnitude between the jet and the cocoon, demonstrating the weakness of the first assumption. The larger effective density of relativistic jets suggests a jet head advance speed nearly that of the jet flow speed (based on the relation for $\beta_{\mathrm{h}}^{*}$ in eq. [A10]), which would violate the assumption of a slowly moving 
jet head. This is confirmed by comparing the average velocities of the terminal shock, $\overline{\beta_{\mathrm{h}}}$ in Table 2 , with $\beta \gtrsim 0.9 c$ for Runs $\mathrm{E}, \mathrm{B}, \mathrm{C}$, and $\mathrm{D}(\beta=0.3$ in Run $\mathrm{A})$. The approximation of a slowly moving head is poorly satisfied for all simulations but Runs A or $\mathrm{A}_{\mathrm{pw}}$. Nevertheless, we see that the predicted cocoon radii are close to those simulated, which suggests that the overall simple model is a valid framework in which to interpret the results.

The analytic expressions for $R_{c} / R$ successfully mimic the typical reduction in cocoon size that accompanies the increase in Lorentz factor in the relativistic runs and for the most part their nonrelativistic equivalents. Primarily because our sets of simulations do not have a large range in estimated $R_{c} / R$, this estimate is not a good discriminate between a relativistic and nonrelativistic flow in our simulations. Nevertheless, we see that either a small $\eta$ with moderate Mach number or a small $\eta_{\mathrm{r}}$ with moderate Lorentz factor is required to generate the typically large cocoon of a FR II source.

\subsection{Jet Head}

Balancing the sum of ram plus thermal pressure at the jet head between the jet and the ambient medium leads to a predicted jet head advance speed, which we will designate

$\beta_{\mathrm{h}}^{*}$ and is equal to $\sqrt{\eta_{\mathrm{r}}} \beta /\left(\sqrt{\eta_{\mathrm{r}}}+1 / \gamma\right)$. In Appendix A, we show a slightly different method for deriving this than given by Martí et al. (1997), clarify the approximations used, and introduce notation used in Appendix B. We define the efficiency as the mean velocity divided by this predicted jet head velocity, i.e. $\bar{\beta}_{\mathrm{h}} / \beta_{\mathrm{h}}^{*}$. We list the advance speeds and the efficiency for each simulation in Table 2, where we have again used inlet velocities to rescale all velocities in the nonrelativistic simulations. From the data in Table 2, it can be seen that the efficiencies in the nonrelativistic equivalents are within $25 \%$ of the efficiencies in the relativistic simulations. Since the only inaccuracy in the position of the terminal shock is the numerical and artificial viscosities that smear the shock over a few zones, the differences in efficiencies between a relativistic and nonrelativistic pair is larger than the error in the measured position.

There is a smaller difference between the efficiencies of these relativistic simulations and those of each run's nonrelativistic equivalent than between the efficiencies of relativistic simulations in Martí et al. (1997) and those of nonrelativistic simulations of cylindrical jets in Norman, Winkler, \& Smarr (1983). The main reason for this smaller difference is that these two sets of simulations have dissimilar initial conditions. Specifically, efficiencies as large as 1.25 in Martí et al. (1997) were associated with highly supersonic, cold jets with an adiabatic index of $4 / 3$, which is unlikely to be equivalent in any sense (e.g., equal in thrust, power, or adiabatic index) to the nonrelativistic simulations in Norman, Winkler, \& 
Smarr (1983) or those presented here. The hot relativistic jets in Martí et al. (1997) have efficiencies near unity, similar to those of our hot relativistic Runs C and D.

Our simulations generally have efficiencies larger than the smallest of those in Norman, Winkler, \& Smarr (1983), who point out that for nonrelativistic jets of equal $\eta$ the efficiency is at a minimum for Mach numbers of roughly 3. The nonrelativistic equivalent simulations have Mach numbers between 6.0 and 25.4, so the efficiencies in our set are understandably larger than the smallest in Norman, Winkler, \& Smarr (1983), which had an efficiency of 0.49. Of our nonrelativistic simulations, Run $\mathrm{A}_{\mathrm{pw}}$ has the smallest Mach number and efficiency of our nonrelativistic simulations, and it is also one of the Norman, Winkler, \& Smarr (1983) set; for the efficiency that we list as 0.61, they find 0.59. Thus, we see that our relativistic simulations have efficiencies smaller than those in Martí et al. (1997), and our nonrelativistic simulations have efficiencies larger than in Norman, Winkler, \& Smarr (1983). Despite the implication of Martí et al. (1997) that only hypersonic (M z 30) nonrelativistic jets have efficiencies similar to those of relativistic jets, we are able to match the efficiencies of relativistic jets with our set of power- or thrust-equivalent supersonic, dense jets.

As with the simulations of Martí et al. (1997), we find that jets with higher Lorentz factors and their nonrelativistic equivalents have greater efficiencies, which is related to the ability of faster, effectively denser jets to have a narrow jet head (see Figure 1). Since the derivation for $\beta_{\mathrm{h}}^{*}$ assumes that the areas on both sides of the terminal shock are equal, the degree of collimation of the jet flow is a primary cause for differences between estimated and computed jet head speeds in any of the simulations. In fact, an explanation for efficiencies greater than unity in the simulations of Martí et al. (1997) is that the softer adiabatic index in a cold cylindrical relativistic jet can focus the jet flow and accelerate the terminal shock. In comparing the efficiencies of Run $\mathrm{C}$ and $\mathrm{D}$, we see that the smaller adiabatic index in a hot relativistic jet does not significantly affect the efficiency.

\subsection{Bow shock}

The useful power and thrust equating cases for Runs C and D display bow shocks that are weaker in the schlieren images than for the other simulations because the jet is transonic with respect to the ambient medium (see $\mathrm{M}_{\mathrm{x}}$ in Table 1 ). Although the strength of the bow shock is different between the relativistic and nonrelativistic simulations, bow shocks are not usually identifiable in extragalactic radio sources. Indeed, the faintness of bow shocks has been suggested by radiative transfer calculations using these relativistic simulations (Mioduszewski, Hughes, \& Duncan 1997). We conclude that this difference cannot be used 
to distinguish relativistic jet flows in actual sources. However, the bow shock can affect the evolution of the cocoon via the shocked ambient medium. For example, a larger $\mathcal{M}$ reduces the Mach angle between the bow shock and the jet axis and leads to greater confinement of the cocoon via an enhanced thermal pressure in the shocked ambient medium.

We list the ages of the jets (in units of a dynamical time, $\mathrm{t}_{\text {dyn }} \equiv \mathrm{R} / \mathrm{a}_{\mathrm{a}}$ ) in Table 2 . In general, the ages for nonrelativistic equivalents are similar to those for the corresponding relativistic simulation. Of particular note is the closeness in ages of the thrust equating cases with the relativistic simulations, which is related to the equivalence of momentum flux and ram pressure, as discussed in $\S 2$. Note that a comparison of ages in dynamical times is more relevant between a relativistic simulation and its nonrelativistic equivalents than within any set of simulations, since the concept of a fiducial "dynamical time" is most useful for cases where $\mathrm{R}$ and $a_{\mathrm{a}}$ can be scaled to the same value in each compared simulation. This cannot be true for this relativistic set of simulations because the relativistic equations require that velocities are not scale-free (and the temperature in the ambient medium is different for each of these relativistic simulations), which is not a requirement of the nonrelativistic simulations. Larger temperatures and sound speeds in the relativistic simulations lead to smaller dynamical times, and explain the larger ages in the hotter and faster relativistic simulations.

\subsection{Internal Structure}

\subsubsection{Comparison of Instability in Relativistic and Nonrelativistic Simulations}

Motivated by the result of HRHD that a larger Lorentz factor is associated with a smaller variation of pressure along the jet axis, we analyze these pressure variations in the nonrelativistic simulations. HRHD pointed out that the variations in Runs A, E, and B are typically larger than those of Runs C and D. Since the thrust-equating nonrelativistic simulations and the relativistic simulations should have similar stability properties (e.g., similar wave speeds and growth lengths, see discussion in $\S 2$ ), we display the pressure variations along an axial slice in both sets of simulations in Figure 5. These slices are taken from a row of zones near $\mathrm{r}=\mathrm{R} / 8$ to avoid uncharacteristically large deviations that occur along the symmetry axis in a cylindrical jet.

A comparison of the axial pressure slices reveals that there are fewer and smaller deviations along the sequence for the nonrelativistic simulations as well. In addition, in the thrust-equating cases we reproduce the fairly abrupt break between many large-amplitude variations in Runs $\mathrm{A}, \mathrm{E}$, and $\mathrm{B}$ and the few small-amplitude variations in Runs $\mathrm{C}$ and D. 
Recall that the analysis in $\S 2$ suggests that the stability properties of Run $B_{\text {th }}$ is most similar to that of Run E of the relativistic set. Since we have grouped the simulations into two groups between Runs B and C, this distinction is not very useful. The trend of fewer and smaller variations in pressure for faster jets is also evident in axial slices of the rescaled axial velocity and to some extent in the rescaled radial velocity, neither of which is shown.

Although we do not display the useful power-equating set of simulations, the set has similar behavior and demonstrates the general trend toward smaller pressure variations as the Lorentz factor is increased in the relativistic set. Thus, the amplitudes of pressure variations within the length of the jet vary similarly in both relativistic simulations and their nonrelativistic equivalents, and the behavior of both sets may readily be understood through linear stability analysis. However, the match between the stability properties of relativistic and nonrelativistic equivalent simulations is less than perfect. Perhaps most importantly, for the nonrelativistic equivalents of the largest Lorentz factor simulations (Runs C and D), variations of the rescaled velocity are larger than the velocity variations in the relativistic simulations because the nonrelativistic simulations have no upper limit on their speed. This difference re-emphasizes the need for fully relativistic simulations.

\subsubsection{Comparison of Instability in Hot and Cold Relativistic Jets}

A detailed stability analysis of Runs A, E, B, C, and D has appeared elsewhere (HRHD) with the conclusion that relativistic jets are not "unconditionally stable" (Martí et al. 1997) but that the simulations which show jets that appear stable have not excited any instabilities. For example, HRHD interpret the apparent relative stability of Run C as due to only a weak coupling of the first body wave of the pinch mode with a pressure wave that originates at the inlet, while the lack of cocoon vorticities prevents any perturbations to the jet surface from jet material that has passed through the terminal shock. Note that this is a common mechanism for exciting the KH instability in supersonic jets.

Ferrari, Trussoni, \& Zaninetti (1978) state that small wavelength perturbations are ineffective for $\gamma>(D / \alpha R)^{1 / 2}$ while large wavelength perturbations are stable, where $\mathrm{D}$ is the length of the jet (e.g., the distance between the parent galaxy and a hot spot) and $\alpha$ is a scaling parameter between 0.01 and 0.1 . This confirms a general result of the stability analysis in HRHD, who found a maximum wavelength for body waves of the pinch mode in a relativistic jet. The condition on $\gamma$ suggests that instabilities can grow for "slow" relativistic jets or within faster jets of sufficient length. For example, this criterion suggests that in our simulations, which terminate when the bow shock reaches $\sim 40 \mathrm{R}$, the jets can show evidence of instability if $\gamma \lesssim 20$ (if $\alpha$ is at its maximum value), which is a condition that all 
of our relativistic jets satisfy. However, as described above, little instability was seen due to the absence of a strong coupling of a KH mode with available perturbations. In comparison, the fastest simulations in Martí et al. (1997) have $\gamma=22.4$ and they were computed out to only 50R, so the jet speed is sufficiently large that very little internal structure formed. Evidently, the condition on $\gamma$ alone is insufficient to assess the stability properties of flows, and careful consideration must also be given to how available perturbations couple with the available modes.

In the set of relativistic simulations, both the bulk flow (the Lorentz factor) and the random motions (the internal pressure) were increased in tandem: the jets in Run A and Run E were cold, the jet in Run B was warmer, and the jets in Runs C and D were hot. One indicator of this is the Mach number (not the relativistic Mach number) in each of these simulations. Since the sound speed within the jets varied from $0.05 c$ to nearly $0.8 c$, the Mach numbers varied between 6 in Run A and 1.28 in Run C. Alternatively, we can calculate the ratio of mass-energy density to the pressure-dependent portion of enthalpy, $(\Gamma-1) n c^{2} /(\Gamma p)$, which is a ratio Bicknell (1994) has designated $\mathcal{R}$. For the $\Gamma=5 / 3$ cases, $\mathcal{R}=1 / 2.5 p$ (with the jet proper density and the speed of light set to unity), which varies by an order of magnitude between each of Runs E, B, and C, with values of 15.6, 1.4, and 0.098, respectively. For Run $\mathrm{D}$ (with $\Gamma=4 / 3$ ), $\mathcal{R}$ is the more familiar $1 / 4 p$ and equal to 0.091. This shows that Run B is the closest to equipartition between mass and thermal energies. Run A is a very cold simulation with $\mathcal{R} \sim 250$.

Our simulations represent an evenly distributed sample over two orders of magnitude in $\mathcal{R}$, and include a simulation near equipartition between mass and thermal energy. By comparison, the simulations in Martí et al. (1997) have $\mathcal{R}$ of either $\mathcal{O}(10)$ or $\mathcal{O}(0.01)$, but nothing in between. Thus, our simulations investigate a new region of parameter space for differences between hot and cold jets.

In order to disentangle the effects of increasing Lorentz factor from those of increasing pressure, we have completed another set of relativistic simulations using the same code and numerical techniques as for the first set. These new simulations fill in places in Lorentz factor-internal pressure $(\gamma-p)$ space beyond Runs E, B, and C (see Table 3). All of the new runs have $\Gamma=5 / 3$. We name the new simulations with the designation of an original simulation with the same Lorentz factor and a subscript indicating a different temperature (e.g., $B_{\text {cool has }} \gamma=5.0$ as did Run B, but a pressure and $\mathcal{R}$ similar to those of the cooler Run E). We also list the relativistic Mach number, $\mathcal{M}$, and the Mach number, $\mathrm{M}$, for each case in Table 3, which shows the decrease in $\mathrm{M}$ that accompanies an increase in thermal pressure.

Schlieren plots of the new set of simulations and Runs E, B, and C are displayed in 
Figure 6, which has columns of equal $p$ increasing to the right and rows of equal $\gamma$ increasing from top to bottom. As with the schlieren plots in Figure 1, all plots in Figure 6 are at the time when the bow shock nears the edge of the grid. We list the age of each simulation in units of a dynamical time in Table 3.

These new simulations have been analyzed for their stability properties, as was done in HRHD. This analysis can explain much of the internal structure in the schlieren images of Figure 6. The normal mode analysis suggests that faster, cooler relativistic jets are more stable, so Run $\mathrm{C}_{\text {cool }}$ should have the least internal structure of the simulations in Figure 6. Any simulation in a panel to the right of (hotter than) or above (slower than) Run $\mathrm{C}_{\text {cool }}$ should be more unstable and have more structure. Hotter simulations should be more unstable because the increased sound speed shortens a jet crossing time for a perturbation. Faster flows are more stable because they naturally increase the spatial growth length. With the exception of the hottest simulations, we do see the expected instability trends, with the slow, hot Run $\mathrm{E}_{\text {warm }}$ appearing the most internally unstable. Therefore, we confirm the most basic results of the stability analysis.

The stability analysis of HRHD also suggests an explanation for the relative lack of

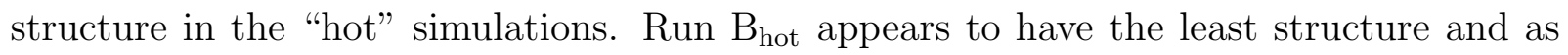
we have already discussed Run $\mathrm{C}$ has only a few small internal variations in pressure. Not only do the conditions in the two hot simulations admit growing modes, as shown by the normal mode analysis, but both have external Mach numbers, $\mathrm{M}_{\mathrm{x}}$, near 1.3 (see Table 3). The stability analysis suggests that this is near a critical value, where the solution to the dispersion relation changes dramatically and quickly. We display pinch mode solutions from the linear analysis for the three simulations with $\gamma=5$, i.e. Runs $\mathrm{B}_{\text {cool }}$, $\mathrm{B}$, and $\mathrm{B}_{\text {hot }}$, in Figure 7. There is a noticeable difference between the shape of the growth rate of the surface wave of the pinch mode for Run $\mathrm{B}$ with $\mathrm{M}_{\mathrm{x}}=1.56$ and for Run $\mathrm{B}_{\text {hot }}$ with $\mathrm{M}_{\mathrm{x}}=$ 1.31, and this difference exists even between Run $C$ with $M_{x}=1.33$ and Run $B_{\text {hot }}$. As the external Mach number decreases toward the critical value of 1.3, the growth rate of the surface wave increases at all frequencies so that it dominates the growth rates of all the body waves, whose solutions remain similar. In addition, the dominant surface wave for $\mathrm{M}_{\mathrm{x}} \lesssim 1.3$ does not pass through a resonance, so there is no frequency associated with a peak growth rate (see Figure 7 ). This contrasts with the body waves, which still have a peak growth rate while the jet is supersonic; body waves are suppressed in a subsonic flow. As with the hot jets in HRHD, the stable appearance of jets in these simulations is most likely due to a weak coupling between the instability at all frequencies and the jet. Additionally, some simple physical arguments for the amplitude of displacement surfaces (e.g., as in HRHD) indicate that short wavelength perturbations will saturate at small amplitudes. These small wavelengths would need adequate grid resolution to grow within a 
numerical simulation.

\section{Comparison with Observations - Cyg A}

We will now apply our results to available observations in the manner of Williams (1991). For the following comparison with observations, we will concern ourselves with data for a single source - Cygnus A. A large fraction of the observations of this source have recently been compiled in a review article by Carilli \& Barthel (1996). At two orders of magnitude above the FR I-FR II break and a redshift of 0.0562 (Stockton, Ridgeway, \& Lilly 1994), Cygnus A is a very powerful and very close double-lobed radio source. Based on results from a 3D MHD nonrelativistic jet simulation with ZEUS-3D, Hardee (1996) suggested that a dense jet with $\gamma \mathrm{M}$, which can be thought of as $\mathcal{M}$ in a cold jet, $\approx 10$ and a jet-to-cocoon density ratio of $\gamma^{2} \eta \approx 6$ is needed in order to reproduce the observed helical and filamentary structure. A dense jet is appropriate for such a simulation since the ambient medium represents the cocoon, which is lighter than the jet.

While Komissarov \& Falle (1998) have modeled Cygnus A with some success by using the self-similar properties of an expanding jet, we apply the results from our cylindrical jets to Cyg A by first estimating the age of the source. The distance of the hotspot or farthest edge of the lobe from the core, $\mathrm{D}$, is $50 \mathrm{~h}_{100}^{-1} \mathrm{kpc}$, where $\mathrm{h}_{100}$ is the Hubble constant normalized by $100 \mathrm{~km} \mathrm{~s}^{-1} \mathrm{Mpc}^{-1}$. We adopt a jet radius $\sim 0.5 \mathrm{kpc}$, based on the results of Perley, Dreher, \& Cowan (1984) who observed that the jet has a constant FWHM of $0{ }^{\prime \prime} 7$ for the inner $1 /{ }_{3} \mathrm{D}$; at the redshift quoted above this implies a radius of $575 \mathrm{~h}_{100}^{-1} \mathrm{pc}$. Therefore the jet has propagated $\sim 100 \mathrm{R}$, and it would take roughly 25-60 dynamical times to reach that distance (based on 10-25 $\mathrm{t}_{\text {dyn }}$ to reach $\sim 40 \mathrm{R}$ in simulations with $\mathcal{M} \sim 8$, see Table 2 ). High resolution ROSAT results indicate the inner $50 \mathrm{kpc}$ of cluster has a temperature of $3 \times 10^{7} \mathrm{~K}$ (Reynolds \& Fabian 1996), so the sound speed in the external medium is roughly $800 \mathrm{~km} \mathrm{~s}^{-1}$ and 25-60 dynamical times corresponds to roughly 15-45 Myrs. This age range straddles the observationally determined value of $30 \mathrm{Myr}$ (Carilli et al. 1991), which is from a self-consistent model based on the spectral aging of the Cyg A lobes. Since the age estimates for the nonrelativistic and relativistic are close, we cannot use this estimate to determine whether the jet in Cyg A propagates at a relativistic speed.

From the analytic expressions for the radius of the cocoon (see eqs. [6] and [7]), we can also estimate $\eta_{\mathrm{r}}$ in the relativistic case or $\eta$ in the nonrelativistic case. The typical radius of the cocoons in Cygnus A is $10 \mathrm{kpc}$, so $\mathrm{R}_{\mathrm{c}} / \mathrm{R}$ is roughly 20. From eq. (6), assuming that the Lorentz factor is in the range of 5-10, and accounting for the fact that the analytic

expression frequently predicted the cocoon center, we estimate an enthalpy ratio of roughly 
$10^{-4}$, with smaller Lorentz factors requiring smaller values of $\eta_{\mathrm{r}}$ (as seen in Figure $3 \mathrm{a}$ ). From eq. (7) and assuming $M$ is roughly 5-10, $\eta$ should be $3 \times 10^{-5}-3 \times 10^{-4}$. This suggestion of a very light jet is consistent with an estimate by Clarke (1996), who from 3D nonrelativistic simulations proposed that $\eta$ is $3 \times 10^{-4}$ and $\mathrm{M}$ is 15 . Either of the estimates for $\eta_{\mathrm{r}}$ and $\eta$ suggest the jet in Cyg A is very light, and possibly cold. Also, it should be noted that this value of $\eta$ is smaller than that of any jet simulation yet attempted.

\section{Conclusions}

In this paper, we have examined morphological and dynamical differences between simulations of relativistic and nonrelativistic jets, including the age, efficiency, velocity field, and internal structure of the jets as well as the size of the cocoon. An important result of these comparisons is that the velocity field of nonrelativistic jet simulations cannot be scaled up to give the spatial distribution of Lorentz factors seen in relativistic simulations. Specifically, such a scaling substantially underpredicts the size of the region of significant Lorentz factor. Since the local Lorentz factor is the primary factor determining the brightness of the source, this suggests that a nonrelativistic simulation cannot yield the proper intensity distribution from a relativistic jet. From the rescaled velocity plots, we see evidence for a result more definitively seen in Mioduszewski, Hughes, \& Duncan (1997): jet flows of moderate Lorentz factor $(\gamma \sim 2-5)$ generate the most knotty appearance.

Other general results are that relativistic simulations and their nonrelativistic equivalents have similar ages (in dynamical time units, $\equiv \mathrm{R} / a_{\mathrm{a}}$ ), efficiencies, and that jets with a larger Lorentz factor have a smaller cocoon size. The ages and efficiencies in the nonrelativistic simulations with equal thrust are a particularly good match to the relativistic simulations. Also, for both relativistic simulations and their nonrelativistic equivalents with $\Gamma=5 / 3$, the widths of the cocoon radii predicted from simple models frequently match a density minimum within the cocoon, and therefore are adequate estimators of cocoon centers. However, due to the limited range of estimated cocoon widths in all of our relativistic and nonrelativistic sets of simulations, these simple models do not discriminate well between relativistic and nonrelativistic flow speeds. Slices of pressure near the symmetry axis vary similarly in both relativistic and nonrelativistic simulation sets. Even in the nonrelativistic simulations, these data slices show fewer and smaller deviations as the Lorentz factor in the equivalent relativistic simulation is increased.

In addition to this comparison, we also have completed four new relativistic simulations to investigate the effect of varying thermal pressure on the structure of simulated relativistic jets. These simulations confirm that faster (larger Lorentz factor), colder jets are more 
stable, with smaller amplitude and longer wavelength internal variations. However, an exception to this occurs for hot jets, where we see evidence beyond that presented in HRHD that hot, relativistic jets appear stable because of a weak coupling between perturbations and available growing KH instability modes. The stability analysis also suggests a different behavior as the external Mach number decreases through $\sim 1$.3. Jets with such a small $\mathrm{M}_{\mathrm{x}}$ should have large growth rates at high frequencies and small wavelengths. These jets appear stable also because of the weak coupling of between the instability and the perturbations within the jet. A higher grid resolution may allow the smaller wavelength perturbations to grow.

As an example of how these simulations can be applied to observations, we use our results to estimate the age, jet-to-ambient density, and jet-to-ambient enthalpy of Cygnus A. Although none of these estimates can determine if the jet in Cyg A is relativistic or nonrelativistic, they confirm independent estimates of these parameters. Our results suggest that the jet is light with $\eta \approx 10^{-4}$, and possibly cold with $\eta_{\mathrm{r}} \approx 10^{-4}$ if the jet has a relativistic flow speed.

Future work will focus on extending the techniques used in the relativistic jet simulations (i.e., AMR and RHLLE) to allow for high resolution three dimensional relativistic simulations and the analysis thereof. Since the growth rates of nonaxisymmetric modes in nonrelativistic jets are larger than the growth rates of the symmetric modes, nonaxisymmetric relativistic jets should be more unstable than the relatively stable simulations presented here. Thus, investigating the stability of $3 \mathrm{D}$ relativistic jets will reveal much about the nature of astrophysical jets.

We thank the Laboratory for Computational Astrophysics for providing the version of ZEUS-3D used for the nonrelativistic simulations, which were run on a Sparc 10 in the Department of Physics and Astronomy at the University of Alabama. New simulations with the DH94 relativistic code were performed on a Power Challenge at the Ohio Supercomputer Center and on an Ultrasparc at the University of Michigan. AR and PEH wish to acknowledge support from the National Science Foundation grants AST-9318397 and AST-9802955 to the University of Alabama and PAH acknowledges support from the National Science Foundation grant AST-9617032 to the University of Michigan. 


\section{A. Head Advance Speed}

The momentum 'discharge' of a jet is

$$
Q=\left[(e+p) u^{2}+p\right] A,
$$

where $e$ is total internal energy density, $p$ is pressure, $u$ is the four-velocity, and $A$ is the area of the flow. An estimate of the advance speed of the head of a jet may be made by balancing the discharges associated with the jet and ambient media in the frame of the advancing Mach disk-contact surface-bow shock structure. Since we assume the area is common, we have

$$
\left(e_{\mathrm{j}}+p_{\mathrm{j}}\right) \gamma_{\mathrm{c}}^{2} \beta_{\mathrm{c}}^{2}+p_{\mathrm{j}}=\left(e_{\mathrm{a}}+p_{\mathrm{a}}\right) \gamma_{\mathrm{a}}^{2} \beta_{\mathrm{a}}^{2}+p_{\mathrm{a}},
$$

where we have used $v / c=u / \gamma=\beta$, and subscript $j$ refers to the jet, while subscript $a$ refers to the ambient medium. Subscript $c$ refers to the jet speed measured in the advancing head frame, so that

$$
\beta_{\mathrm{c}}=\frac{\beta_{\mathrm{j}}-\beta_{\mathrm{a}}}{1-\beta_{\mathrm{j}} \beta_{\mathrm{a}}},
$$

and

$$
\gamma_{\mathrm{c}}=\left(1-\beta_{\mathrm{c}}^{2}\right)^{-1 / 2} .
$$

If the jet and ambient pressures are equal, or the jet flow is hypersonic, the above simplifies to

$$
\left(e_{\mathrm{j}}+p_{\mathrm{j}}\right) \gamma_{\mathrm{c}}^{2} \beta_{\mathrm{c}}^{2}=\left(e_{\mathrm{a}}+p_{\mathrm{a}}\right) \gamma_{\mathrm{a}}^{2} \beta_{\mathrm{a}}^{2} .
$$

Making the following definitions:

$$
\begin{gathered}
e=\varepsilon \rho, \\
\eta=\frac{\rho_{\mathrm{j}}}{\rho_{\mathrm{a}}}<1,
\end{gathered}
$$

and

$$
\eta_{\mathrm{r}}=\eta \frac{\varepsilon_{\mathrm{j}}+\frac{p_{\mathrm{j}}}{\rho_{\mathrm{j}}}}{\varepsilon_{\mathrm{a}}+\frac{p_{\mathrm{a}}}{\rho_{\mathrm{a}}}},
$$

we see that

$$
\eta_{\mathrm{r}} \gamma_{\mathrm{c}}^{2} \beta_{\mathrm{c}}^{2}=\gamma_{\mathrm{a}}^{2} \beta_{\mathrm{a}}^{2} .
$$

Substituting for $\beta_{\mathrm{c}}$ and $\gamma_{\mathrm{c}}$ from equations (A3) and (A4), after some algebra we find

$$
\beta_{\mathrm{a}}=\frac{\sqrt{\eta_{\mathrm{r}}} \beta_{\mathrm{j}}}{\sqrt{\eta_{\mathrm{r}}}+\gamma_{\mathrm{j}}^{-1}},
$$

which is the speed of ambient flow towards the head, and thus the speed of the head through the ambient medium. Note that the quantity $\beta_{\mathrm{a}}$ is the predicted head velocity, which is 
designated $\beta_{\mathrm{h}}^{*}$ in the text. This is the relativistic generalization of the familiar Newtonian form

$$
v_{\mathrm{h}}=\frac{v_{\mathrm{j}}}{1+\eta^{-1 / 2}}
$$

\section{B. Cocoon Radius}

\section{B.1. Relativistic Formulation}

The particle 'discharge' of a jet is

$$
P=n v \gamma A
$$

where $n$ is the baryon density, and other quantities are as defined above. Jet material thermalized at a Mach disk expands laterally to from a cocoon, but the particle discharge is conserved, so

$$
n_{\mathrm{j}} v_{\mathrm{j}} \gamma_{\mathrm{j}} A_{\mathrm{j}}=n_{\mathrm{c}} v_{\mathrm{c}} \gamma_{\mathrm{c}} A_{\mathrm{c}}
$$

where the subscript $c$ refers to the cocoon. As $A_{\mathrm{j}}=\pi R_{\mathrm{j}}^{2}$ and $A_{\mathrm{c}}=\pi R_{\mathrm{c}}^{2}-\pi R_{\mathrm{j}}^{2}$ (the jet displaces cocoon material), we have

$$
\left(\frac{R_{\mathrm{c}}}{R_{\mathrm{j}}}\right)^{2}=1+\frac{n_{\mathrm{j}} v_{\mathrm{j}} \gamma_{\mathrm{j}}}{n_{\mathrm{c}} v_{\mathrm{c}} \gamma_{\mathrm{c}}}
$$

If we adopt the picture that shocked jet material streams beyond the Mach disk to the vicinity of the contact surface, and expands laterally to form the cocoon, then, until further longitudinal expansion has given the material a back flow velocity in the frame of the head, this material is moving at the speed of the head - as characterized by $\beta_{\mathrm{a}}$ and $\gamma_{\mathrm{a}}$ above. Thus

$$
\left(\frac{R_{\mathrm{c}}}{R_{\mathrm{j}}}\right)^{2}=1+\frac{n_{\mathrm{j}}}{n_{\mathrm{h}}} \frac{n_{\mathrm{h}}}{n_{\mathrm{c}}} \frac{v_{\mathrm{j}}}{v_{\mathrm{a}}} \frac{\gamma_{\mathrm{j}}}{\gamma_{\mathrm{a}}}
$$

We may write

$$
\frac{n_{\mathrm{h}}}{n_{\mathrm{c}}}=\left(\frac{p_{\mathrm{h}}}{p_{\mathrm{c}}}\right)^{1 / \Gamma}=\left(\frac{p_{\mathrm{h}}}{p_{\mathrm{j}}}\right)^{1 / \Gamma},
$$

if the jet is in pressure balance with the cocoon, so that $p_{\mathrm{c}}=p_{\mathrm{j}}$. Using the previously-derived relation for $v_{\mathrm{a}}$, the term

$$
\frac{v_{\mathrm{j}}}{v_{\mathrm{a}}} \frac{\gamma_{\mathrm{j}}}{\gamma_{\mathrm{a}}}
$$

may be recast, and after some algebra we get

$$
\left(\frac{R_{\mathrm{c}}}{R_{\mathrm{j}}}\right)^{2}=1+\left(\frac{p_{\mathrm{h}}}{p_{\mathrm{j}}}\right)^{1 / \Gamma} \frac{n_{\mathrm{j}}}{n_{\mathrm{h}}}\left(\frac{1+\eta_{\mathrm{r}}+2 \sqrt{\eta_{\mathrm{r}}} \gamma_{\mathrm{j}}}{\eta_{\mathrm{r}}}\right)^{1 / 2} .
$$




\section{B.2. Relativistic Jump Values}

The terms $p_{\mathrm{h}} / p_{\mathrm{j}}$ and $n_{\mathrm{j}} / n_{\mathrm{h}}$ are determined by the Rankine-Hugoniot conditions at the Mach disk. The simplest case (applicable to Run D in DH94) is for $\Gamma=4 / 3$; then these

ratios are functions only of $\gamma_{\mathrm{j}}$. Let primes denote values measured in the frame of the shock discontinuity, and subscript 1 signify the upstream state. Given the extreme equation of state, from Königl (1980) we have

$$
\frac{p_{\mathrm{h}}}{p_{\mathrm{j}}}=\frac{2 \hat{\Gamma}_{1} \mathcal{M}_{1}^{2}-\left(\hat{\Gamma}_{1}-1\right)}{\hat{\Gamma}_{1}+1}=\frac{4 \mathcal{M}_{1}^{2}-1}{3}=\frac{8 \gamma_{\mathrm{j}}^{\prime 2} \beta_{\mathrm{j}}^{\prime 2}-1}{3},
$$

because

$$
\hat{\Gamma}=\gamma_{\text {sound }}^{2} \Gamma=\frac{3}{2} \times \frac{4}{3}=2
$$

and

$$
\mathcal{M}_{1}=\frac{\gamma_{1}^{\prime} \beta_{1}^{\prime}}{\left.\gamma^{\prime} \beta^{\prime}\right|_{\text {sound }}}=\frac{\gamma_{1}^{\prime} \beta_{1}^{\prime}}{1 / \sqrt{2}}
$$

for the adopted adiabatic index. From mass conservation

$$
n_{1} \beta_{1}^{\prime} \gamma_{1}^{\prime}=n_{2} \beta_{2}^{\prime} \gamma_{2}^{\prime}
$$

so that

$$
\frac{n_{\mathrm{j}}}{n_{\mathrm{h}}}=\frac{\beta_{\mathrm{h}}^{\prime} \gamma_{\mathrm{h}}^{\prime}}{\beta_{\mathrm{j}}^{\prime} \gamma_{\mathrm{j}}^{\prime}}
$$

For the adopted adiabatic index, $\beta_{1}^{\prime} \beta_{2}^{\prime}=1 / 3$ (for any flow speed), and after some algebra the above equation may be recast as

$$
\frac{n_{\mathrm{j}}}{n_{\mathrm{h}}}=\frac{\gamma_{\mathrm{j}}^{\prime}}{\left(8 \gamma_{\mathrm{j}}^{\prime 4}-17 \gamma_{\mathrm{j}}^{\prime 2}+9\right)^{1 / 2}}
$$

Note that both pressure and density depend on primed quantities - those measured in the head frame. For situations in which the head moves slowly compared with the jet flow speed, it will be a reasonable approximation to use the jet parameters directly.

\section{B.3. Nonrelativistic Formulation}

Using arguments similar to those above we have

$$
\rho_{\mathrm{j}} v_{\mathrm{j}} A_{\mathrm{j}}=\rho_{\mathrm{c}} v_{\mathrm{c}} A_{\mathrm{c}}
$$


so that

$$
\left(\frac{R_{\mathrm{c}}}{R_{\mathrm{j}}}\right)^{2}=1+\frac{v_{\mathrm{j}}}{v_{\mathrm{c}}} \frac{\rho_{\mathrm{j}}}{\rho_{\mathrm{c}}}
$$

It follows as before that

$$
\left(\frac{R_{\mathrm{c}}}{R_{\mathrm{j}}}\right)^{2}=1+\left(1+\eta^{-1 / 2}\right) \frac{\rho_{\mathrm{j}}}{\rho_{\mathrm{h}}}\left(\frac{p_{\mathrm{h}}}{p_{\mathrm{j}}}\right)^{1 / \Gamma}
$$

From the nonrelativistic Rankine-Hugoniot conditions we may write

$$
\frac{\rho_{\mathrm{j}}}{\rho_{\mathrm{h}}}\left(\frac{p_{\mathrm{h}}}{p_{\mathrm{j}}}\right)^{1 / \Gamma}=\left[\frac{(\Gamma-1) \mathrm{M}^{2}+2}{(\Gamma+1) \mathrm{M}^{2}}\right] \times\left[\frac{2 \Gamma \mathrm{M}^{2}-(\Gamma-1)}{(\Gamma+1)}\right]^{1 / \Gamma}
$$

Note again that Mach number is determined by the upstream flow speed in a frame moving with the shock, but that for a fast jet and slowly moving bow, the jet speed will yield a good approximation to this. 


\section{REFERENCES}

Bicknell, G.V. 1994, ApJ, 422, 542

Bodo, G., Massaglia, S., Ferrari, A., \& Trussoni, 1994, A\&A, 283, 655

Bodo, G., Massaglia, S., Rossi, P., Rosner, R., Malagoli, A., \& Ferrari, A. 1995, A\&A, 303, 281

Carilli, C.L., \& Barthel, P.D. 1996, A\&A Rev., 7, 1

Carilli, C.L., Perley, R.A., Dreher, J.W., \& Leahy, J.P. 1991, ApJ, 383, 554

Cawthorne, T.V. 1991, in Beams and Jets in Astrophysics, ed. P.A. Hughes (Cambridge: Cambridge Univ. Press), 187

Clarke, D.A. 1996, in Energy Transport in Radio Galaxies and Quasars, ed. P.E. Hardee, A.H. Bridle, \& J.A. Zensus (San Francisco: Astronomical Society of the Pacific), 311

Duncan, G.C., \& Hughes, P.A. 1994, ApJ, 436, L119

Duncan, C., Hughes, P., \& Opperman, J. 1996, in Energy Transport in Radio Galaxies and Quasars, ed. P.E. Hardee, A.H. Bridle, \& J.A. Zensus (San Francisco: Astronomical Society of the Pacific), 143

Einfeldt, B. 1988, SIAM J. Numer. Analy., 25, 294

Falle, S.A.E.G., \& Komissarov, S.S. 1996, MNRAS, 278, 586

Ferrari, A., Trussoni, E., \& Zaninetti, L. 1978, A\&A, 64, 43

Hardee, P.E. 1987, ApJ, 318, 78

- 1996, in Cygnus A — Study of a Radio Galaxy, ed. C.L. Carilli \& D.E. Harris (Cambridge: Cambridge Univ. Press), 113

Hardee, P.E., Clarke, D.A., \& Howell, D.A. 1995, ApJ, 441, 644

Hardee, P.E., \& Norman, M.L. 1988, ApJ, 334, 70

Hardee, P.E., Rosen, A., Hughes, P.A., \& Duncan, C.G. 1998, ApJ, 500, 598 (HRHD)

Koide, S., Nishikawa, K.-I., \& Mutel, R.L. 1996, ApJ, 463, L71

Koide, S., Shibata, K., \& Kudoh, T. 1998, ApJ, 495, L63 
Komissarov, S.S., \& Falle, S.A.E.G. 1996, in Energy Transport in Radio Galaxies and Quasars, ed. P.E. Hardee, A.H. Bridle, \& J.A. Zensus (San Francisco: Astronomical Society of the Pacific), 173

Komissarov, S.S., \& Falle, S.A.E.G. 1997, MNRAS, 288, 833

Komissarov, S.S., \& Falle, S.A.E.G. 1998, MNRAS, 297, 1087

Königl, A. 1980, Phys. Fluids, 23, 1083

Martí, J.Mä., Müller, E., Font, J.A, \& Ibáñez, J.Mㅁ․ 1995, ApJ, 448, L105

Martí, J.Mํ.., Müller, E., Font, J.A, \& Ibáñez, J.Mํㅡ., \& Marquina, A. 1997, ApJ, 479, 151

Martí, J.Mํ․, Müller, E., \& Ibáñez, J.Mํㅡ. 1994, A\&A, 281, L9

Mioduszewski, A.J., Hughes, P.A., \& Duncan, G.C. 1997, ApJ, 476, 649

Norman, M.L. 1996, in Energy Transport in Radio Galaxies and Quasars, ed. P.E. Hardee, A.H. Bridle, \& J.A. Zensus (San Francisco: Astronomical Society of the Pacific), 405

Norman, M.L., \& Winkler, K.-H.A. 1985, Los Alamos Science, 11, 38

Norman, M.L., Winkler, K.-H.A., \& Smarr, L. 1983, in Astrophysical Jets, ed. A. Ferrari \& A.G. Pacholczyk (Dordrecht: Reidel), 227

Norman, M.L., Winkler, K.-H.A., \& Smarr, L. 1984, in Physics of Energy Transport in Extragalactic Radio Sources, ed. A.H. Bridle \& J.A. Eilek (NRAO Proceedings, No. 9), 150

Reynolds, C., \& Fabian, A. 1996, MNRAS, 278, 479

Stockton, A., Ridgeway, S.E., \& Lilly, S.J. 1994, AJ, 108, 414

Quirk, J.J. 1991, Ph.D. Thesis, Cranfield Institute of Technology

van Putten, H.P.M. 1996, ApJ, 467, L57

Williams, A.G. 1991, in Beams and Jets in Astrophysics, ed. P.A. Hughes (Cambridge: Cambridge Univ. Press), 342 
Fig. 1.- Schlieren plots of the relativistic and both corresponding nonrelativistic sets of simulations. Darker shading indicates a larger gradient in the density in the nonrelativistic sets or lab frame density in the relativistic set. Each set of five vertical panels shows Runs A, E, B, C, and D or their equivalents. Scales shown along each axis are in units of jet radius, R. Each panel contains an arrow showing the axial position of radial profiles in Figure 4.

Fig. 2.- Contours of low and high Lorentz factors. We show a low and high contour of $\gamma$ for the relativistic (Runs A, E, B, C, and D) and of rescaled $\gamma$ in the useful power (Runs $\mathrm{A}_{\mathrm{pw}}, \mathrm{E}_{\mathrm{pw}}$, $\mathrm{B}_{\mathrm{pw}}, \mathrm{C}_{\mathrm{pw}}$, and $\mathrm{D}_{\mathrm{pw}}$ ) simulations. The rescaling of velocity in the nonrelativistic simulations is described in §3.1.1. Note that the plots for runs $\mathrm{C}$ and $\mathrm{D}$ and their nonrelativistic equivalents have been cropped to show only the inner 10R on the radial axis.

Fig. 3.- Estimates of cocoon radius in $\eta_{\mathrm{r}}-\gamma$ or $\eta-\mathrm{M}$ space. The contours of equal cocoon radius, which are labeled by $R_{c} / R$, are from the equations in Appendix B. In panel a), the region at the upper left (above the jagged contour) corresponds to a subsonic flow and no cocoon is allowed by the model. The placement of each simulation in either panel is determined by the input jet data listed in Table 1 .

Fig. 4.- Radial slices of density and pressure for all 14 simulations, taken at the axial position of minimum density. The solid line shows the density, $\rho$, for the nonrelativistic simulations or the lab frame density, $\nu$, for the relativistic simulations, the dotted line shows the pressure, and the dashed line shows the proper density, $n$, in the relativistic simulations. The vertical line in each panel is at the radius given by eq. (6) for relativistic Runs B-E, or eq. (B7) in all other cases. The vertical axis is the same for each row of panels, but not each column.

Fig. 5.- Slices parallel to the axis of jet pressure relative to the inlet jet pressure, $p_{\mathrm{b}}$. The logarithm of pressure is shown for a row of zones near but not on the jet axis for the relativistic (solid line) jet centered on $r=5 / 48 \mathrm{R}$, which is the middle of the third zone, and thrust-equating nonrelativistic (dashed line) jet on $r=3 / 24 \mathrm{R}$, which is the middle of the second zone. Since the highest spatial resolution in the relativistic simulations is 24 zones/R and the resolution in the nonrelativistic simulations is 12 zones/R, these two rows overlap in physical space.

Fig. 6. - Schlieren plots of the relativistic simulations that can be compared to show the effect of temperature (hot vs. cold jets). The top row has $\gamma=2.5$, the middle one has $\gamma=$ 5 , and the bottom one has $\gamma=10$. Each column differs in pressure by roughly an order of magnitude compared to an adjacent column (increasing left to right). 
Fig. 7.- Real (dotted lines) and imaginary (dashed lines) part of the wavenumber as a function of frequency from linear stability analysis for the $\gamma=5$ simulations. For Runs $\mathrm{B}_{\text {cool }}, \mathrm{B}$, and $\mathrm{B}_{\text {hot }}$, we display the solutions for the surface, labeled $\mathrm{S}$, and first two body waves, labeled $\mathrm{B}_{1}$ and $\mathrm{B}_{2}$, of the pinch mode. Note the different behavior for the surface wave in Run $B_{\text {hot }}$. 
Table 1. Simulation Parameters

\begin{tabular}{|c|c|c|c|c|c|c|c|c|c|c|c|}
\hline \multirow[b]{2}{*}{$\operatorname{Run}^{\mathrm{a}}$} & \multirow[b]{2}{*}{$\Gamma$} & \multirow[b]{2}{*}{$\eta_{\mathrm{r}}$} & \multirow[b]{2}{*}{$\mathcal{M}$} & \multirow[b]{2}{*}{$\gamma$} & \multicolumn{3}{|c|}{ Power } & \multicolumn{3}{|c|}{ Thrust } & \multirow{2}{*}{$\frac{\text { Stability }}{\eta_{\mathrm{x}}{ }^{\mathrm{c}}}$} \\
\hline & & & & & $\eta$ & M & $\mathrm{M}_{\mathrm{a}}$ & $\eta$ & M & $\mathrm{M}_{\mathrm{a}}$ & \\
\hline $\mathrm{A}^{\mathrm{b}}$ & $5 / 3$ & 0.10 & 6 & 1.05 & 0.11 & 6.1 & 18.1 & 0.11 & 6.0 & 18.1 & \\
\hline $\mathrm{E}$ & $5 / 3$ & 0.11 & 8 & 2.5 & 1.03 & 10.2 & 9.9 & 0.70 & 8.3 & 9.9 & 8.8 \\
\hline B & $5 / 3$ & 0.16 & 8 & 5.0 & 6.70 & 12.6 & 4.9 & 3.96 & 9.4 & 4.7 & 41.8 \\
\hline $\mathrm{C}$ & $5 / 3$ & 0.56 & 8 & 10.0 & 73.1 & 18.0 & 2.1 & 55.5 & 12.8 & 1.7 & 83.0 \\
\hline $\mathrm{D}$ & $4 / 3$ & 0.57 & 15 & 10.0 & 74.4 & 25.4 & 2.9 & 57.1 & 18.0 & 2.4 & 93.1 \\
\hline
\end{tabular}

a Relativistic simulations A-D were originally discussed in DH94, Run E was added for HRHD.

${ }^{\mathrm{b}} \mathrm{A}$ single nonrelativistic equivalent of this slow jet was run with $\eta=0.1, \mathrm{M}=$ 6.0 and $\mathrm{M}_{a}=19.0$.

${ }^{c}$ Estimated from setting the wave speed of the maximum growing modes (equation [5]) to its nonrelativistic equivalent with the appropriate values from the relativistic simulations. 
Table 2. Simulation Data

\begin{tabular}{llrccc}
\hline \hline Run & $\beta_{\mathrm{s}, \mathrm{a}}$ & $\mathrm{t}_{\mathrm{dyn}}$ & $\bar{\beta}_{\mathrm{h}}$ & $\beta_{\mathrm{h}}^{*}$ & Efficiency \\
& & & & & \\
\hline $\mathrm{A}$ & 0.017 & 11.0 & 0.058 & 0.075 & 0.77 \\
$\mathrm{~A}_{\mathrm{pw}}$ & & 14.0 & 0.044 & 0.072 & 0.61 \\
$\mathrm{E}$ & 0.092 & 8.1 & 0.314 & 0.417 & 0.75 \\
$\mathrm{E}_{\mathrm{pw}}$ & & 9.8 & 0.433 & 0.464 & 0.93 \\
$\mathrm{E}_{\mathrm{th}}$ & & 8.5 & 0.373 & 0.419 & 0.89 \\
$\mathrm{~B}$ & 0.208 & 14.7 & 0.554 & 0.652 & 0.85 \\
$\mathrm{~B}_{\mathrm{pw}}$ & & 12.3 & 0.646 & 0.707 & 0.91 \\
$\mathrm{~B}_{\mathrm{th}}$ & & 14.5 & 0.575 & 0.654 & 0.88 \\
$\mathrm{C}$ & 0.581 & 26.9 & 0.828 & 0.877 & 0.94 \\
$\mathrm{C}_{\mathrm{pw}}$ & & 21.5 & 0.868 & 0.891 & 0.97 \\
$\mathrm{C}_{\mathrm{th}}$ & & 26.5 & 0.840 & 0.876 & 0.96 \\
$\mathrm{D}$ & 0.418 & 19.5 & 0.845 & 0.879 & 0.96 \\
$\mathrm{D}_{\mathrm{pw}}$ & & 15.5 & 0.876 & 0.891 & 0.98 \\
$\mathrm{D}_{\mathrm{th}}$ & & 19.4 & 0.852 & 0.878 & 0.97 \\
\hline
\end{tabular}


Table 3. Hot vs. Cold $(\Gamma=5 / 3)$ Jet Simulation Data

\begin{tabular}{lcccrrcrcc}
\hline \hline Run & $\mathrm{p}$ & $\beta_{\mathrm{s}}$ & $\mathcal{R}$ & $\gamma$ & $\mathcal{M}$ & $\mathrm{M}$ & $\mathrm{t}_{\text {dyn }}\left(\mathrm{R} / a_{\mathrm{a}}\right)^{\mathrm{a}}$ & $\mathrm{M}_{\mathrm{x}}$ \\
& & & & & & & & \\
\hline $\mathrm{E}$ & 0.051 & 0.275 & 7.8 & 2.5 & 8.0 & 3.33 & 11.5 & 2.86 \\
$\mathrm{~B}_{\text {cool }}$ & 0.051 & 0.275 & 7.8 & 5.0 & 17.1 & 3.56 & 5.7 & 1.96 \\
$\mathrm{C}_{\text {cool }}$ & 0.051 & 0.275 & 7.8 & 10.0 & 34.8 & 3.62 & 4.6 & 1.88 \\
$\mathrm{E}_{\text {warm }}$ & 0.277 & 0.522 & 1.4 & 2.5 & 3.7 & 1.76 & 24.1 & 1.67 \\
$\mathrm{~B}$ & 0.277 & 0.522 & 1.4 & 5.0 & 8.0 & 1.88 & 14.7 & 1.56 \\
$\mathrm{C}_{\text {warm }}$ & 0.277 & 0.522 & 1.4 & 10.0 & 16.3 & 1.91 & 10.5 & 1.63 \\
$\mathrm{~B}_{\text {hot }}$ & 4.096 & 0.779 & 0.1 & 5.0 & 3.9 & 1.26 & 30.8 & 1.31 \\
$\mathrm{C}$ & 4.095 & 0.779 & 0.1 & 10.0 & 8.0 & 1.28 & 26.9 & 1.33 \\
\hline
\end{tabular}

${ }^{a}$ Dynamical times of jets simulations at the time of the image shown in Figure 6. 
This figure "rhdhfig1.gif" is available in "gif" format from: http://arxiv.org/ps/astro-ph/9901046v1 

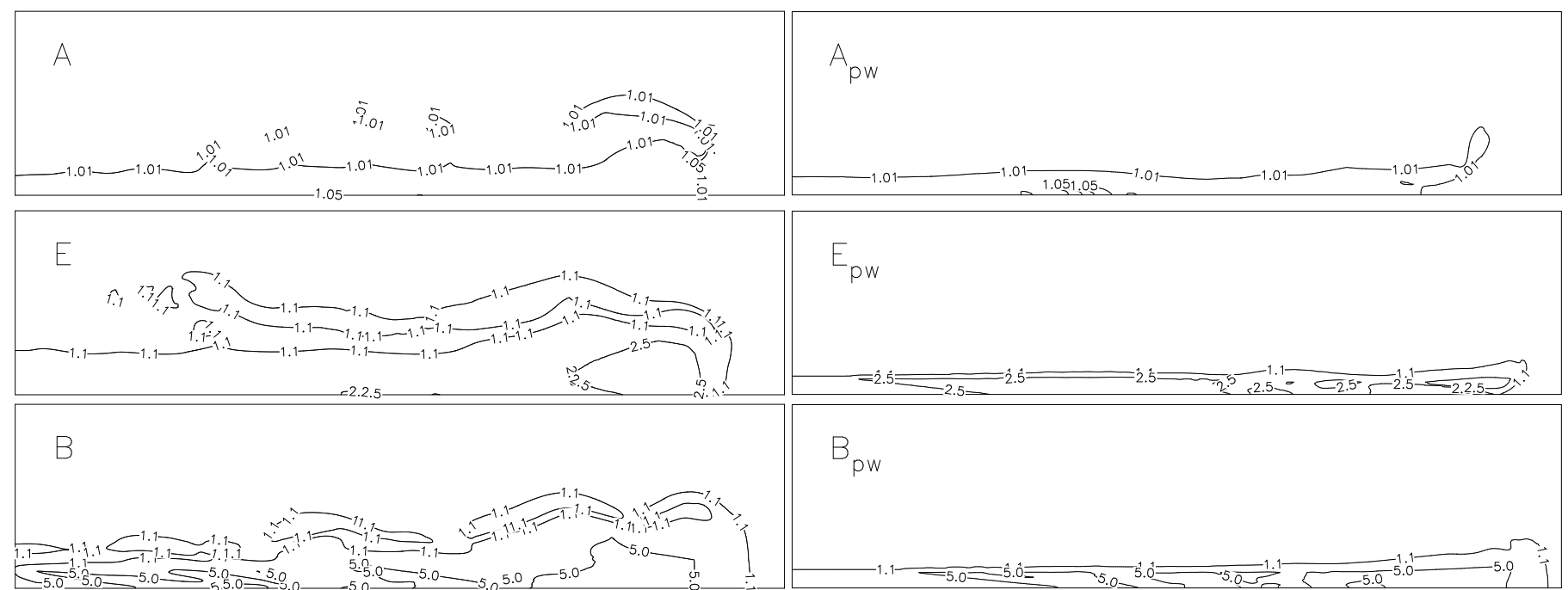

B pw
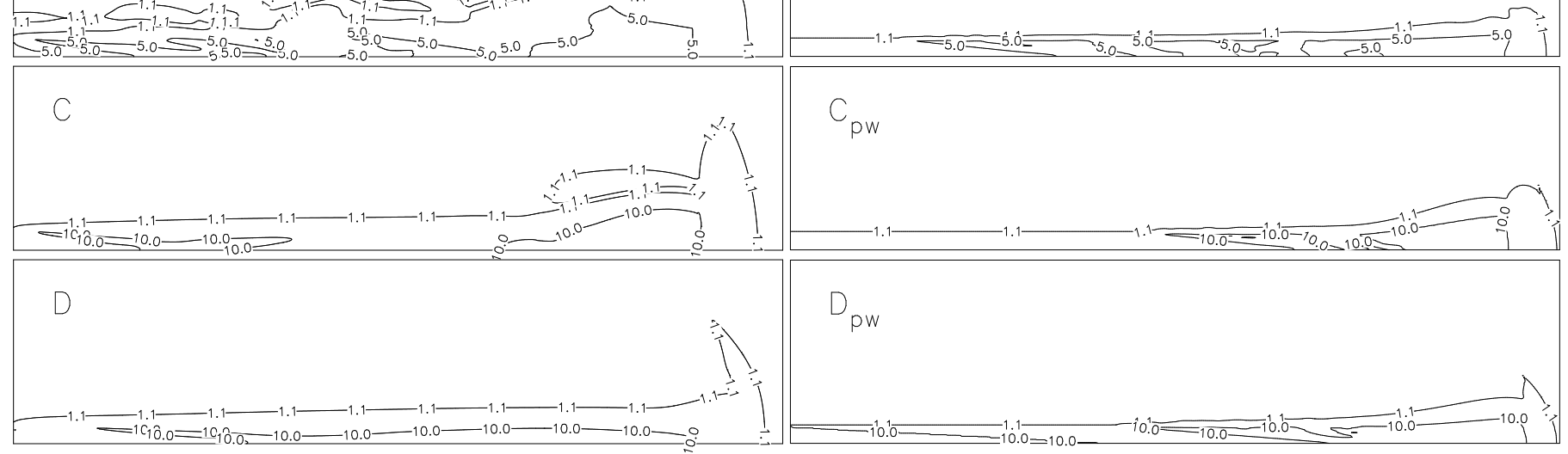

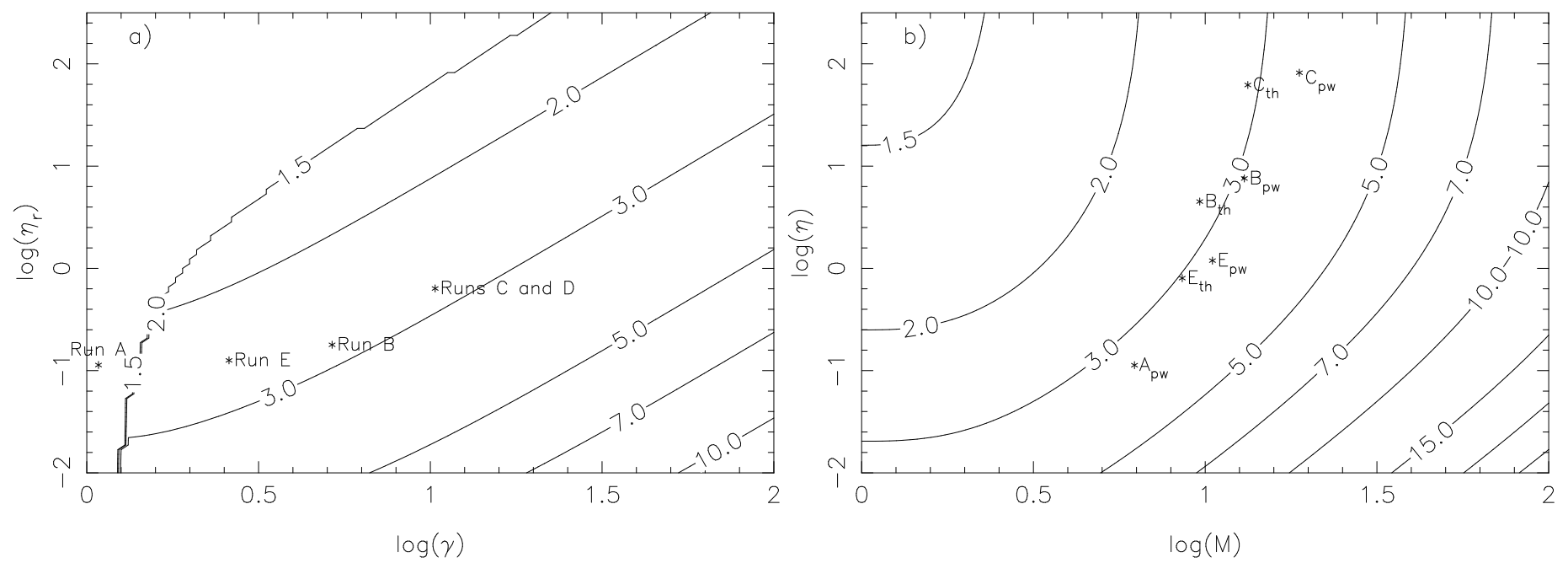

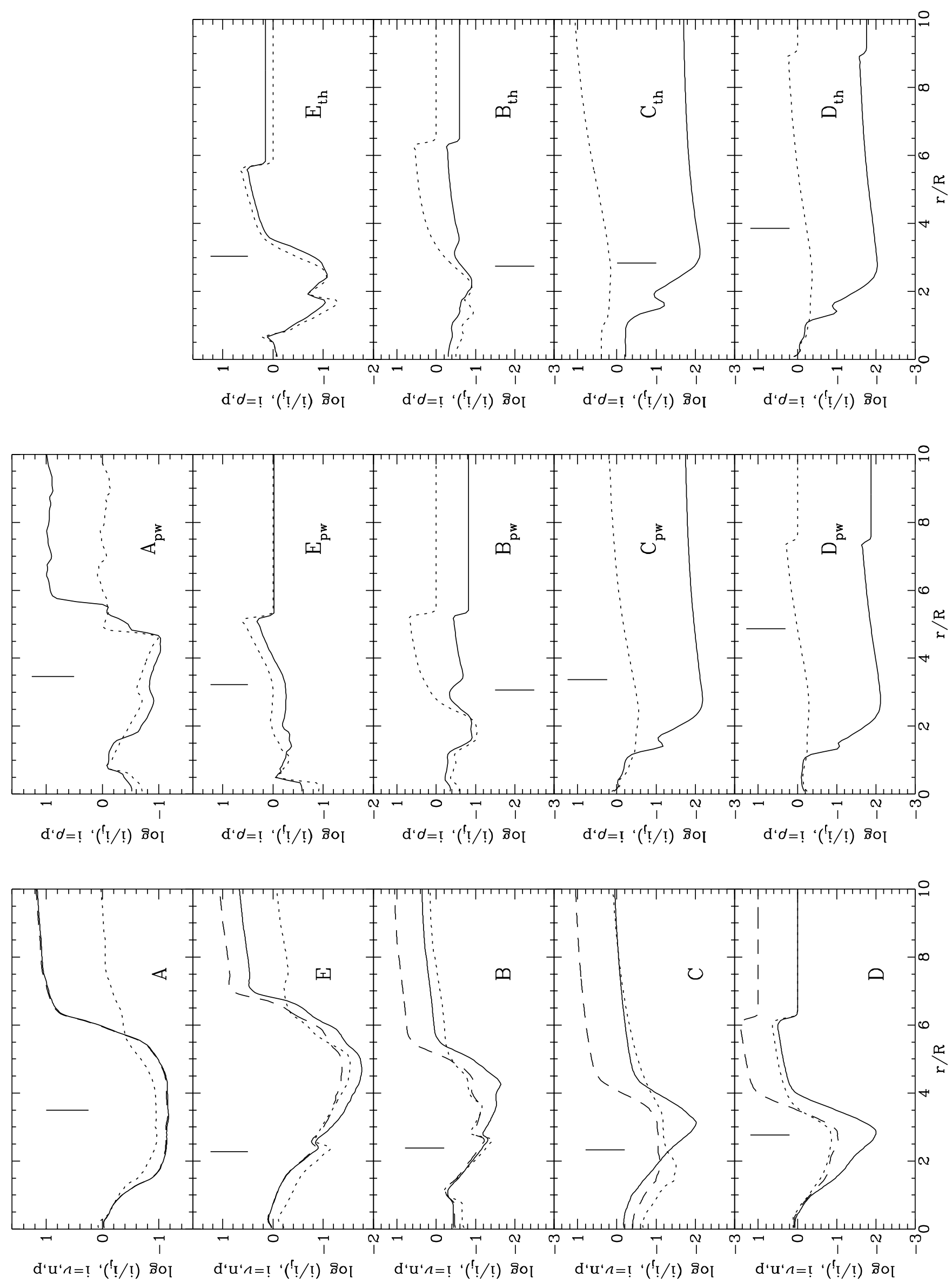


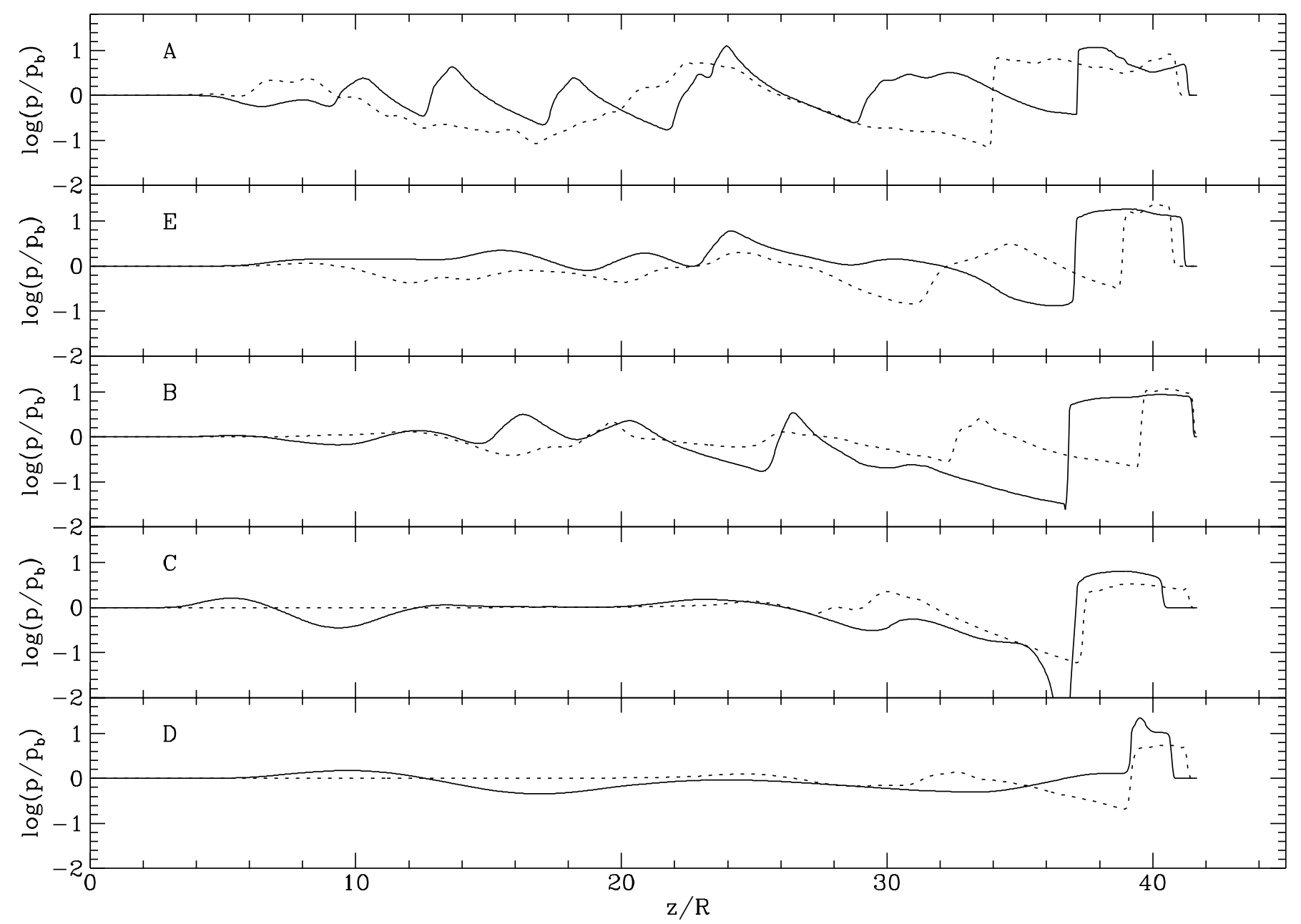


This figure "rhdhfig6.gif" is available in "gif" format from: http://arxiv.org/ps/astro-ph/9901046v1 


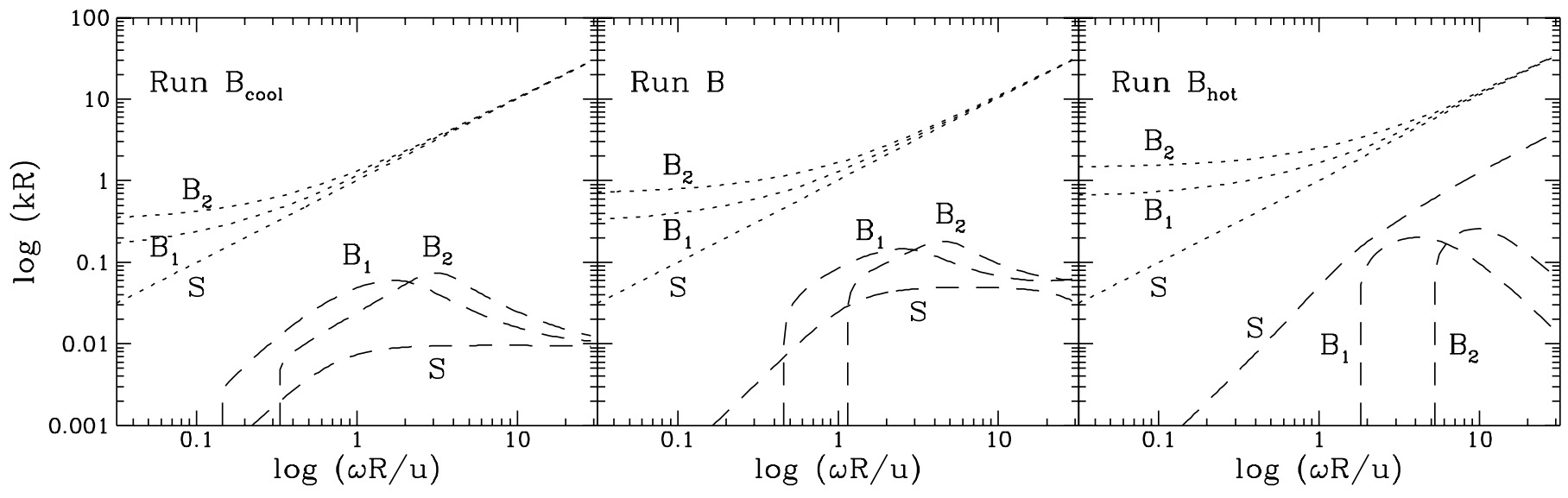

